\title{
Sistemas de información para la gestión de mantenimiento en la gran industria del estado Zulia *
}

\author{
Oliva, Karim ** \\ Arellano, Madelein *** \\ López, María **** \\ Soler, Karen ${ }^{* * * * *}$
}

\section{Resumen}

Este trabajo tiene como propósito determinar las características que distinguen los sistemas de información para la gestión de mantenimiento en la gran industria del estado Zulia. Este estudio está basado en una entrevista estructurada aplicada a los gerentes, jefes o encargados de mantenimiento a catorce (14) industrias de la región seleccionadas de una población de treinta y cuatro (34) empresas, mediante la cual se determinó la condición de los elementos constitutivos de los sistemas de información para mantenimiento a saber, administración de los equipos, órdenes de trabajo, especialidades de mantenimiento, abastecimiento y control de materiales, informes de desempeño y tecnologías de información utilizadas. En los resultados se evidencia que la gestión de mantenimiento en las industrias encuestadas hace énfasis en la administración de los equipos y en la utilización de las órdenes de trabajo como mecanismo de planificación y de control de las actividades de mantenimiento. Se observa heterogeneidad en las características de los sistemas de información que apoyan la gestión de mantenimiento, debido principalmente al empleo disímil de las tecnologías de información. Se concluye que las industrias con mayor nivel de automatización

Recibido: 21-01-09. Aceptado: 10-12-09

* Este trabajo constituye un avance del Proyecto de Investigación titulado Información para la Gestión Privada, financiado por El Consejo de Desarrollo Científico y Humanístico (CONDESLUZ) y adscrito al Centro de Estudios de la Empresa.

** Investigadora-Docente de la Facultad de Ingeniería. E-mail: karimoliva@gmail.com

*** Investigadora-Docente de la Facultad de Ciencias Económicas y Sociales. Cursante del Doctorado en Ciencias Sociales, mención Gerencia. E-mail: marellano@luz.edu.ve. Ingeniera industrial con maestría en Gerencia de Empresas de la Universidad del Zulia y adscritas al Programa de promoción al investigador del FONACIT.

**** Ingeniera industrial con maestría en Gerencia de Empresas de la Universidad del Zulia y adscritas al Programa de promoción al investigador del FONACIT.

E-mail: marivi260281@gmail.com

***** Ingeniera Industrial. E-mail: karenssissy@gmail.com 
Sistemas de información para la gestión de mantenimiento en la gran industria del Zulia Oliva, Karim; Arellano, Madelein; López, María y Soler, Karen

muestran sistemas de información más organizados y con mejores posibilidades para el control de la gestión de mantenimiento.

Palabras clave: Sistema de información, gran industria, gestión de mantenimiento, estado Zulia.

\title{
Information Systems for Managing Maintenance in the Main Industry of the State of Zulia
}

\begin{abstract}
The purpose of this work is to determine the characteristics that distinguish information systems for managing maintenance in the principal industry in the State of Zulia. The study is based on a structured interview applied to the managers, heads or those in charge of maintenance for fourteen (14) industries in the region selected from a population of thirty-four (34) companies. The study determined the condition of the constitutive elements for maintenance information systems, such as equipment administration, work orders, maintenance specialties, material storage and control, performance reports and information technologies used. Results show that maintenance management in the interviewed industries emphasizes equipment administration and the use of work orders as planning and control mechanisms for maintenance activities. Heterogeneity was observed in the information system characteristics that support maintenance management, due principally to dissimilar use of information technologies. Conclusions are that industries with a greater level of automation evidence more organized information systems with greater possibilities for controlling maintenance management.
\end{abstract}

Key words: Information system, main industry, maintenance management, State of Zulia.

\section{Introducción}

Para cualquier organización industrial el mantenimiento es considerado un factor estratégico en la búsqueda de incrementar los niveles de productividad, calidad y seguridad en una empresa. El desempeño de un sistema de mantenimiento implica un constante y eficiente manejo de información, lo cual permite a la gerencia integrar las acciones de la empresa durante el desarrollo de los procedimientos y actividades correspondientes a su entorno productivo.

La presente investigación ha sido realizada tomando como base una revisión bibliográfica sobre la concepción de sistemas de información (SI), enfocando su aplicación a la función de manteni- miento en grandes industrias. El propósito es determinar las características que distinguen los sistemas de información para la gestión de mantenimiento en la gran industria del estado Zulia, unificando criterios sobre la estructura y el funcionamiento de los sistemas de información, como elemento fundamental de soporte para el desempeño de la función de mantenimiento. De esta manera, se contrasta esta caracterización con evidencia empírica en las grandes industrias del estado Zulia.

La gran industria está conformada por grandes cantidades de capital y de personas, siendo un factor potencial de cambio para el desarrollo futuro del sector económico y diversos beneficios que otorgan a la sociedad. Particularmente, la 
gran industria posee mayores fortalezas desde la perspectiva de disponibilidad de recursos y organización, respecto a la pequeña y mediana industria.

Ante la dinámica de un mercado cambiante y exigente, las industrias se encuentran sometidas a enormes presiones para ser competitivas y ofrecer una entrega oportuna de productos con calidad, lo cual requiere la implantación de un sistema productivo acorde a estas exigencias y se hace imprescindible la utilización y modernización de equipos con cierta tecnología para el proceso de transformación.

Por ello, el uso de nuevas tecnologías para optimizar los procesos de producción, automatización y distribución son pilar fundamental para este sector industrial; dado que, por lo general, estas empresas cuentan con tecnologías y sistemas de punta que les permiten evaluar distintos escenarios y tomar decisiones, de una manera que les ahorra tiempo y dinero ${ }^{1}$.

Considerando que no hay un criterio único para definir una gran industria, se adoptó el criterio establecido por el Instituto Nacional de Estadística (I.N.E), que considera como gran industria aquella que tenga en su haber un número mayor a cien (100) empleados. Con este criterio, se determinó la población de las grandes industrias ubicadas en el estado
Zulia, totalizando para el año 2007 una cantidad de 34 industrias. La muestra estuvo conformada por 14 empresas, seleccionadas mediante la técnica de muestreo aleatorio simple ${ }^{2}$, que representan el $41.17 \%$ de la población. En las industrias seleccionadas, se aplicó una entrevista estructurada mediante un cuestionario a gerentes, jefes o encargados del área de mantenimiento de cada industria, con el fin de obtener evidencia empírica y acercamiento al objeto de estudio relacionado con las actividades de mantenimiento.

\section{Sistemas de información en las organizaciones}

Un sistema de información "acepta recursos de datos como entrada y los procesa en resultados de información como salida, la cual, a su vez, está constituida por "datos convertidos a un contexto significativo y útil para usuarios finales específicos" (O’Brien, 2001: 42). Particularmente, Goldratt (1994: 5-6) enfatiza que la información sólo se puede definir dentro del marco de la toma de decisiones, estando este proceso imbricado en el sistema de información. De allí, que se plasme que el contexto y el usuario van a determinar el proceso de decisión, propio de la gestión de cualquier tipo de organización y relacionado con su sistema de información.

1 Según cifras aportadas por el Centro Nacional de Tecnologías de Información (C.N.T.I.), el desarrollo industrial venezolano se expresa en aproximadamente un $10 \%$ de establecimientos de la gran industria.

2 El cálculo fue realizado considerando la fórmula de poblaciones finitas establecida por Sierra Bravo (1997:227), asumiendo $40 \%$ y $60 \%$ para p y q, respectivamente, con un nivel de confianza de $95 \%(2 \sigma)$ y error de $20 \%$. 
Sistemas de información para la gestión de mantenimiento en la gran industria del Zulia Oliva, Karim; Arellano, Madelein; López, María y Soler, Karen

Ahora bien, para Oz (2000: 527) en el plano de la planeación estratégica se requiere la declaración de una misión corporativa que aclara su propósito y sus metas generales, y que proporcionan un marco referencial para la formulación de los objetivos estratégicos de la organización. "La misión delinea el propósito de los sistemas de información en la organización"; de esta manera, vincula la estrategia con el sistema de información, determinando el tipo de organización y sus responsabilidades.

En el ámbito de la organización, Burch y Grudnitski (1998: 583) consideran dos filosofías relacionadas con la utilización de SI: "(a) Reactiva, orientada a la base de la organización, y visualiza el sistema de información como un arma defensiva táctica y operacional que responde al procesamiento de datos y reportes; y (b) Proactiva, orientada hacia toda la organización, donde el SI representa un arma ofensiva estratégica. La primera permite que la organización se mantenga y sobreviva, la segunda ofrece una ventaja competitiva".

Por su parte, Montilva (1999: 55) refiere que el sistema de información de una organización "representa un subsistema, que a su vez está conformado por diferentes subsistemas de información estrechamente interrelacionados, asociados a las áreas funcionales, y que como un todo contribuyen a alcanzar los objetivos organizacionales".

La configuración de los sistemas de información se presenta con aspectos distintivos entre diversos autores, relacionados con: (a) grado de especificidad, (b) alcance y (c) clasificación de los elementos que los configuran. Por ello, se analizarán algunas tipologías, para identificar los elementos propios de los sistemas de información.

Según Oz (2001:16) todos los sistemas de información operan de la misma forma, mediante cuatro operaciones principales: "Introducción de datos en el sistema de información (entrada), cambio y manipulación de los datos en el sistema de información (procesamiento de datos), obtención de información fuera del sistema de información (salida) y almacenamiento de datos e información (almacenamiento)". Así, establece una configuración básica que se encuentra relacionada con las funciones de un sistema de información, más no así con los elementos constitutivos del mismo.

Por su parte, Cohen y Asín (2005: 7-8) refieren que los sistemas de información comprenden cuatro actividades básicas: a) entrada de información, proceso mediante el cual el sistema de información toma los datos que requiere para procesar la información; b) almacenamiento de información, definida como la capacidad del sistema para guardar información; c) procesamiento de información, relacionado con la capacidad del sistema de información para efectuar cálculos, que permite la transformación de datos fuente en información que puede ser utilizada para la toma de decisiones; $y$ d) salida de información, visto como el producto del sistema de información y relacionado con su capacidad para emitir información procesada o bien datos de entrada al exterior. Éstos se corresponden con los procesos principales de todo sistema de información, sin obviar una función de retroalimentación, que el sistema debe cumplir como mecanismo para 
ejercer control sobre aquellos procedimientos a los cuales soporta el sistema de información.

Por su parte, Burch y Grudnitski (1998: 58-61,64,66) conforman los sistemas de información en seis componentes estructurales, a saber: a) entrada, esquemas que le permiten al usuario comunicarse con el sistema mediante una combinación de preguntas y respuestas, comandos, teclas de función, menús, entre otros; b) modelos, los cuales utilizan la entrada y datos almacenados, para producir los resultados deseados; c) salida, como el producto del sistema de información, con características de calidad y plasmada en documentos para todos tipo de usuario; d) tecnología, la cual reduce el tiempo y el espacio entre el usuario y el sistema, y está conformada por: computadora y almacenamiento auxiliar, telecomunicaciones y software; e) base de datos, lugar en donde se almacenan todos los datos necesarios para atender a las necesidades de los usuarios; y, finalmente, f) controles, que proporcionan a los usuarios un sistema confiable y el tipo de acceso que necesiten para realizar sus tareas o sostener un diálogo con el sistema.

Para Cohen y Asín (2005: 7) los elementos que conforman un sistema de información incluyen: a) equipo computacional, computadoras o cualquier otro dispositivo (hardware) necesario para que el sistema de información pueda operar; b) recurso humano, refiere aquellas personas que interactúan con el sistema de información; c) datos o información fuente, aquello que se introduce en el sistema para generar como resultado la información que desea; d) programas, aquellos ejecutados por las computadoras, que hace que los datos de entrada introducidos sean procesados y generen los resultados que se esperan; e) telecomunicaciones, que a su vez se conforman de equipo computacional y programas, pero específicamente relacionados con aquellos que facilitan la transmisión de texto, datos, imágenes y voz en forma electrónica; y f) procedimientos, que incluyen las políticas y reglas de operación, tanto en la parte funcional del proceso de negocio, como los mecanismos para hacer trabajar una aplicación en la computadora.

Estas tipologías difieren significativamente, dado que Burch y Grudnitski incluyen la entrada y salida como componentes, cuando realmente se identifican como procesos del sistema de información; además su identificación de las bases de datos como un lugar de almacenamiento se contrapone a lo planteado por $\mathrm{Oz}$ cuando identifica el almacenamiento como una operación de los sistemas de información. Por otra parte, los componentes designados como modelos y controles, se corresponderían, en un sentido más amplio, con la definición de procedimientos dada por Cohen y Asín. Así, coincidimos con Burch y Grudnitski, sólo en la designación de tecnologías como un elemento de los sistemas de información.

El contraste entre estos planteamientos, radica en ver las tecnologías como un elemento de los sistemas de información y no que debido a sus potencialidades, las TIC se presenten excediendo el desarrollo y funcionalidad del sistema de información.

Los aportes de Burch y Grudnitski (1998: 63-64) permiten visualizar la tecnología informática como un factor de interconexión que forma parte de la infraes- 
Sistemas de información para la gestión de mantenimiento en la gran industria del Zulia Oliva, Karim; Arellano, Madelein; López, María y Soler, Karen

tructura de una organización para coordinar sus operaciones. Esto permite que los sistemas no estén funcional y espacialmente separados del lugar de trabajo, sino que se presenten como una red interconectada de trabajo.

En este caso, partiremos del concepto de tecnologías de información planteado por Barros (1998:11-12), quien las identifica con la denominación moderna dada al conjunto de elementos computacionales ${ }^{3}$ en el cual se instalan aplicaciones ${ }^{4}$, las cuales permiten coordinar esos elementos y dirigir su funcionamiento para generar un determinado servicio a un usuario final. Se incluyen adicionalmente las comunicaciones por medio de redes, capaces de interconectar computadores y aplicaciones 5 .

Tal como lo plantea Carr (2005: 12) "la importancia estratégica de las tecnologías de información y comunicación (TIC) no es creciente, como muchos afirman o suponen, sino decreciente. A medida que éstas llegan a ser más poderosas, más estandarizadas y más accesibles, dejan de ser tecnologías propietarias que las compañías pueden usar como una ventaja sobre sus rivales, para convertirse en tecnologías infraestructurales que todos los competidores comparten". Las TIC se convierten en un factor de producción que permite a las organizaciones mantenerse en el mercado, pero no asegura su sobrevivencia.

Barros (1998:16) asevera que "el papel de la tecnología está totalmente determinado por cómo se realicen las actividades humanas del sistema -en cuanto a métodos, prácticas, técnicas y procedimientos-" y su ejecución está determinada por las posibilidades de las tecnologías. Por ello, de acuerdo a lo planteado por Cohen y Asín, otro de los elementos que conforman un SI son los procedimientos. Así, Barros plantea una inseparabilidad entre cómo hacer las actividades humanas y el uso las tecnologías de información, por ello, se establece como fundamental la organización administrativa del quehacer humano, y su registro permitirá realizar una adecuación de los procedimientos al promover alguna iniciativa de automatización. Asumimos que los otros dos elementos constitutivos de un SI son: las personas que interactúan con él y los datos, partiendo de la definición de Goldratt en cuanto a que la información se conforma como resultado o producto del sistema.

3 Se corresponde con el hardware o componentes físicos - monitor, CPU, memoria principal (RAM), disco duro, periféricos, etc. - el computador en sí.

4 Compuesto por el software - sistema operativo o programas de aplicación -, o sea, las instrucciones en lenguaje computacional.

5 Barros refiere cualquier otro componente como parte de una aplicación computacional, lo cual parece ambiguo. Asumimos los elementos computacionales como tecnología dura (hardware) y las aplicaciones como tecnologías blandas (software), cualquier otro componente, exceptuando los comunicacionales, pertenece a estas categorías. 


\section{Gestión de mantenimiento en la gran industria}

El funcionamiento de un equipo origina un desgaste, que en el tiempo produce efectos en su operatividad ocasionando interrupciones al proceso productivo, desde el incumplimiento de compromisos adquiridos o escasez, inclusive hasta perjuicios legales. También un mantenimiento adecuado contribuye a minimizar riesgos de trabajo. Por ello, el funcionamiento de los equipos es una garantía para las exigencias de producción y depende, principalmente, de un adecuado sistema de mantenimiento, ya sea con base en un sistema de información tradicional o un sistema acorde a los avances tecnológicos de esta era informacional.

El mantenimiento es un servicio que agrupa una serie de actividades mediante las cuales un equipo, máquina, construcción civil o instalación, se mantiene o se restablece a un estado apto para realizar sus funciones, siendo importante en la calidad de los productos y como estrategia para una competencia exitosa.

Los esfuerzos realizados en el área de mantenimiento consisten en reducir al mínimo el efecto de las averías de los equipos y de las instalaciones, y proporcionar información relacionada con la experiencia y conocimiento a los departamentos relacionados con el proceso productivo, a fin de reducir el trabajo de mantenimiento, el tiempo improductivo y sus costos.

Para Dounce (1984) el objetivo básico del mantenimiento es maximizar la disponibilidad de los equipos e instalaciones para las actividades de producción, y para ello propone los siguientes objetivos específicos: a) asegurar el funcionamiento confiable y eficiente de los equipos e instalaciones, acorde a los patrones establecidos de seguridad, protección ambiental e imagen de la empresa; b) evitar el deterioro acelerado de los equipos e instalaciones; c) proporcionar los servicios necesarios para la continuidad operacional de las instalaciones, y el bienestar de la comunidad.

Entre las funciones de mantenimiento destaca la diferenciación de tres grandes grupos: administrativas, técnicas y de operación. Las administrativas comprenden: (a) política y organización del mantenimiento, (b) planificación y programación de las actividades de mantenimiento, (c) análisis y evaluación económica y de presupuesto, (d) necesidades y capacitación del personal, (e) registro de subcontratistas, (f) registros e informes para el control de mantenimiento, (g) control de partes de repuestos y de herramientas.

Las funciones técnicas están referidas a: (a) análisis de rendimiento de los equipos, (b) estudio de las causas de fallas, (c) preparación de normas e instrucciones de inspección, exploración, reparación y análisis de sustituciones. Finalmente, en las funciones de operación se incluyen: (a) la inspección (rutinaria, periódica y de aceptación), (b) operaciones de preparación (lubricación, ajuste y reparación), (c) trabajos de ingeniería (maquinado, soldadura, acabado, entre otros) ${ }^{6}$.

El cumplimiento de estas funciones implica la aplicación de criterios y métodos innovadores, tanto en el área de la ingeniería como en el área de informática. En consecuencia, y en concordancia con lo expresado por García (1998), el diseño 
Sistemas de información para la gestión de mantenimiento en la gran industria del Zulia Oliva, Karim; Arellano, Madelein; López, María y Soler, Karen

de la organización y los procesos de mantenimiento deben estar soportados por un sistema de gestión de la información que posibilite la incorporación de modificaciones derivadas de la innovación tecnológica, y la integración de información con la perspectiva de una gestión global de la organización.

Por ello, se requiere una clara definición de los procesos asociados al mantenimiento, identificando responsabilidades y perfiles de acceso a la información adecuados que garanticen tanto la seguridad del sistema como la pertinencia de la información introducida y emitida.

Duffuaa, Raouf y Dixon (2000:302303), establecen dos elementos fundamentales para el funcionamiento eficaz de un sistema computarizado de administración del mantenimiento: "(a) capacidad para apoyar las principales actividades en el proceso del mantenimiento, (b) capacidad de configuración del software y el hardware en términos de su confiabilidad, facilidad de uso, calidad de la información y procesamiento oportuno".

Al incrementarse la capacidad de procesamiento de la información, los sistemas de información en mantenimiento han evolucionado para utilizar las nuevas tecnologías que generan ciertos beneficios. De esta manera, García (1998) afirma que algunos de los beneficios derivados incluyen: a) la reducción de costos y el aumento de la eficacia de la función de mantenimiento; b) mejora de la fiabilidad y disponibilidad y alargamiento del ciclo de vida de las instalaciones; c) aumento de la seguridad integral; d) reducción de stocks en la gestión de aprovisionamientos; y e) mejora en la disponibilidad de información adecuada sobre mantenimiento para la toma de decisiones empresariales.

Si bien la incorporación al área de mantenimiento de un sistema asistido por computadora no es un requisito indispensable para lograr una gestión de mantenimiento eficaz, su implementación facilita y simplifica significativamente dicha gestión cambiando el funcionamiento de las organizaciones. Las organizaciones deben estar dispuestas a una comprensión de los sistemas de información y aprehender una cultura informática para el desarrollo de este tipo de proyectos.

\section{Caracterización de los sistemas de información para mantenimiento}

La investigación de campo para la recopilación de la evidencia empírica relacionada con las actividades de mantenimiento se estructuró con base en las características específicas de los sistemas de información en la función de mantenimiento planteadas por Duffuaa, Raouf y Dixon (2000: 303). Estas características comprenden la información básica que debe poseer un sistema computarizado de información para mantenimiento, estableciendo cinco módulos: 1) administración del equipo; 2) control de órdenes de trabajo; 3) administración de las especiali-

6 Las actividades técnicas y operativas subyacen al control administrativo, por ello, la evidencia empírica se recopilará tomando como base las actividades administrativas. 
dades de mantenimiento; 4) abastecimiento y control de materiales; e 5) informes de desempeño ${ }^{7}$. Para esta caracterización se incluyó el elemento de tecnologías de información como factor primordial en los sistemas de información.

Cabe destacar que todas las empresas encuestadas poseen en su estructura organizativa la función de mantenimiento como una función diferenciada de carácter operativo, lo cual implica que esta función es establecida como una gerencia o un departamento con responsabilidades y recursos específicos.

El análisis estará enfocado particularmente hacia el control de la gestión de mantenimiento, dada la trascendencia que posee esta función para las actividades de producción, el cual requiere de un sistema ágil y coordinado de captura y procesamiento de información que permita la toma de decisiones oportunas.

a. Administración del equipo

La mejora de la eficacia del equipo requiere la medición de las pérdidas que afectan la disponibilidad del equipo, su eficiencia y la calidad del producto. Para Duffuaa, Raouf y Dixon (2000) la información básica que se debe disponer sobre los equipos es su identificación (normalmente asociada a un código), ubicación, fecha de instalación, estado o condición, información técnica, historia del equipo (incluye fallas registradas, tipo de fallas) y programa de mantenimiento, herramien- tas especiales y procedimientos de seguridad.

En la Tabla 1 se aprecia la relevancia que las industrias del sector dan a sus activos; se refleja un alto grado de organización en cuanto a la disposición de archivos de información codificada y clasificada sobre la identificación y las características técnicas de los equipos; sin embargo, los sistemas de información reflejan debilidad en los registros del historial de fallas de los equipos y en la existencia de mecanismos que permitan determinar la condición operativa de los equipos, la cual es una información vital para la toma de decisiones. Se presenta, también, deficiencia en la identificación del equipo con respecto a: a) su ubicación, que puede ser en aquellos equipos con facilidad para su movilización y no se realiza su respectivo rastreo, y b) fecha de fabricación, que para efectos de depreciación del equipo puede ser considerado como elemento fundamental.

\section{b. Órdenes de trabajo (ODT's)}

El propósito del sistema de órdenes de trabajo es proporcionar medios para solicitar por escrito el trabajo que va a realizar el departamento de mantenimiento, seleccionar por operación el trabajo solicitado, asignar el mejor método y los trabajadores más calificados para el trabajo, reducir el costo mediante una utilización eficaz de los recursos, mejorar la planeación y la programación del trabajo

7 Para efectos de esta investigación se consideran estos módulos relacionados con los Datos de un sistema de información, necesarios para la producción pertinente de información y el control de gestión del mantenimiento. No se realizará énfasis en los elementos de Personas y Procedimientos, dada la delimitación del tema. 
Sistemas de información para la gestión de mantenimiento en la gran industria del Zulia Oliva, Karim; Arellano, Madelein; López, María y Soler, Karen

Tabla 1. Administración del equipo

\begin{tabular}{lc}
\hline \multicolumn{1}{c}{ Característica } & Porcentaje \\
\hline $\begin{array}{l}\text { Existencia de base de datos } \\
\text { de los equipos }\end{array}$ & $100 \%$ \\
\hline $\begin{array}{l}\text { Información contenida } \\
\text { en la base de datos: }\end{array}$ & \\
- Código & $100 \%$ \\
- Especificaciones & $92,9 \%$ \\
- Número de serie & $85,7 \%$ \\
- Fabricante & $85,7 \%$ \\
- Ubicación & $57,1 \%$ \\
- Fecha de fabricación & $57,1 \%$ \\
Existencia de historial de vida & \\
de los equipos & $57,1 \%$ \\
$\begin{array}{l}\text { Existencia de criterios de } \\
\text { jerarquización de los equipos }\end{array}$ & $100 \%$ \\
$\begin{array}{l}\text { Existencia de archivos de infor- } \\
\text { mación técnica de los equipos }\end{array}$ & $100 \%$ \\
$\begin{array}{l}\text { El sistema suministra informa- } \\
\text { ción sobre la condición del }\end{array}$ & \\
equipo en cualquier momento & $28,6 \%$ \\
\hline
\end{tabular}

Fuente: Elaboración propia.

de mantenimiento, y mejorar el mantenimiento en general. Dependiendo del diseño del formato de la ODT, ésta puede ser utilizada como una solicitud de trabajo, un documento de planeación, un registro histórico, una herramienta para el monitoreo y control, y una notificación de trabajo completado.

En cuanto a la existencia de las ODT's, como puede observarse en la Tabla 2 , se hace un uso significativo de este mecanismo de planeación y de control para los trabajos de mantenimiento; sin embargo, aún persisten industrias que no lo utilizan, empleando otros procedimientos poco formales.

La información contenida en las ODT's de las grandes industrias encues-
Tabla 2. Órdenes de trabajo

\begin{tabular}{lc}
\hline \multicolumn{1}{c}{ Característica } & Porcentaje \\
\hline $\begin{array}{l}\text { Existencia de formatos } \\
\text { de ODT }\end{array}$ & $78,6 \%$ \\
\hline $\begin{array}{l}\text { Información contenida } \\
\text { en la ODT: }\end{array}$ \\
- Tiempo consumido en el \\
$\quad$ trabajo \\
- Tiempo muerto \\
- Fecha y hora de culminación \\
$\quad$ de los trabajos \\
- Causa de las fallas \\
- Consecuencia de las fallas \\
- Materiales utilizados \\
- Herramientas utilizadas
\end{tabular}

Fuente: Elaboración propia.

tadas está dirigida principalmente a conocer el impacto de las fallas en la producción y al uso de los recursos de mantenimiento; sin embargo, a este respecto se presentan deficiencias en cuanto al control de materiales y herramientas utilizadas.

c. Administración de las especialidades de mantenimiento

El seguimiento a las especialidades de mantenimiento resulta crucial para suministrar a la unidad de planificación la información necesaria para programar las ODT's. El sistema de información debe contener información referente a: nombre del personal, fecha de contratación, especialidad, salario, cargo, código de la especialidad, estado de la especialidad (asignación, vacaciones, médi$\mathrm{co}$, en servicio), entre otras.

Los resultados del estudio muestran una escasa inclinación hacia el registro de la información de los trabajadores por parte de la gestión de mantenimiento, ya que esta responsabilidad se considera 
propia del departamento de recursos humanos, lo cual desde el punto de vista del manejo de la información para la planificación y el control de las actividades de mantenimiento representa una gran debilidad, dado que de la administración apropiada de este recurso depende en gran medida la eficiencia de la gestión de mantenimiento.

Tabla 3. Administración de las especialidades de mantenimiento

\begin{tabular}{lc}
\hline \multicolumn{1}{c}{ Característica } & Porcentaje \\
\hline $\begin{array}{l}\text { Existencia de base de datos } \\
\text { de los trabajadores. }\end{array}$ & $35,7 \%$ \\
\hline \multicolumn{2}{l}{ El sistema permite el registro } \\
de la condición de los \\
trabajadores en cualquier \\
momento. \\
\hline $\begin{array}{l}\text { Información registrada del } \\
\text { personal de mantenimiento: }\end{array}$ \\
- Especialidad \\
- Cargo \\
- Dirección \\
- Años de servicio \\
- Fecha de contratación \\
- Grado de instrucción \\
- Salario \\
\hline
\end{tabular}

Fuente: Elaboración propia.

\section{d. Abastecimiento y control de materiales}

Un funcionamiento adecuado del sistema de mantenimiento depende en gran medida de la administración de los inventarios de materiales. De hecho, una parte significativa del presupuesto de mantenimiento está representado por este rubro, lo cual induce a la consideración de factores económicos. Si la inversión es excesiva los resultados son elevados costos de mantenimiento de inventario $^{8}$. Por el contrario, si no se cuenta con las refacciones y materiales necesarios para las reparaciones y servicios de los equipos, el costo de tiempo muerto se puede incrementar enormemente. Por ello, los esfuerzos deben estar dirigidos a lograr un sistema de administración de inventarios equilibrado.

En el proceso de elaboración de una ODT, el planificador identifica los materiales que se requerirán, para lo cual debe verificar que se cuente con las existencias necesarias. Duffuaa, Raouf y Dixon (2000: 311) son contundentes al expresar que "la disponibilidad de los materiales ${ }^{9}$ es decisiva para una planeación, programación y control sin contratiempos de los trabajos de mantenimiento".

8 En general, las empresas tienden a reducir sus inventarios, realizando esfuerzos en cuanto a sus costos operacionales con menores cantidades de pedido y trabajando estrechamente con sus proveedores. Así, se disminuyen tiempos de suministro y se reduce la necesidad de mantener elevados inventarios (Davis; Aquilano y Chase, 2001: 465), básicamente, porque el costo de mantenimiento de inventario varía en proporción directa con el nivel de inventarios.

9 Esto puede lograrse con la interconexión a las funciones básicas de un sistema de información de inventario que muestre: disponibilidad de los materiales, materiales solicitados y su estado, órdenes de compra abiertas, ajustes de inventario, elaboración de órdenes de compra, entre otras. 
Sistemas de información para la gestión de mantenimiento en la gran industria del Zulia Oliva, Karim; Arellano, Madelein; López, María y Soler, Karen.

En la Tabla 4 se observa que la condición de los sistemas de información en relación a la gestión de los materiales de mantenimiento es precaria en la mayoría de las industrias encuestadas. La disponibilidad de información sobre materiales recae en el departamento de compras o en el almacén de materiales ( $50 \%$ de las industrias), mientras que el sistema de información de mantenimiento no cuenta con una base de datos interrelacionada al sistema de inventario.

En relación a la información que se registra en la base de datos, es significativo el hecho de que en apenas el $42,9 \%$ de las industrias, la función de mantenimiento maneja información sobre el precio de los materiales; esto obedece principalmente a que las decisiones de compra normalmente las toma otro departamento. Asimismo, la clasificación de materiales para las decisiones de las políticas de inventario, tampoco es considerada relevante en la gestión de mantenimiento.

En pocas industrias $(21,4 \%)$, el sistema de información de mantenimiento puede proporcionar información en tiempo real sobre la condición del inventario, información fundamental para la programación de las ODT's. Esta deficiencia de información dificulta la verificación de existencias de repuestos en momentos críticos para la operatividad de las plantas.

La disposición de un directorio de proveedores de servicios de mantenimiento y fabricantes que contenga su identificación y desempeño permite asegurar las mejores decisiones de contratación; de manera similar, la selección de proveedores de materiales requiere la disponibilidad de esta información. Sólo el $35,7 \%$ de los sistemas de información de
Tabla 4. Abastecimiento y control de materiales

\begin{tabular}{lc}
\hline \multicolumn{1}{c}{ Característica } & Porcentaje \\
\hline $\begin{array}{l}\text { Existencia de base de datos } \\
\text { de materiales y repuestos } \\
\text { para mantenimiento. }\end{array}$ & \\
\hline $\begin{array}{l}\text { Información registrada en la } \\
\text { base de datos: }\end{array}$ \\
- Código \\
- Descripción del material \\
$\quad$ o repuesto \\
- Proveedor \\
- Cantidad en existencia \\
- Precio \\
- Clasificación \\
$\begin{array}{l}\text { El sistema permite la consulta } \\
\text { de la condición del inventario }\end{array}$ \\
en cualquier momento. \\
$\begin{array}{l}\text { El sistema registra información } \\
\text { sobre los proveedores. }\end{array}$ \\
\hline
\end{tabular}

Fuente: Elaboración propia.

mantenimiento de las industrias encuestadas poseen estos registros actualizados.

Se observa una gran vulnerabilidad en este elemento que es primordial para el buen desempeño de la gestión de mantenimiento, particularmente en aquellas empresas que no poseen sistemas de información integrados, y que no tienen la posibilidad de manejar la información de disponibilidad de inventario en tiempo real. Debe existir una relación muy estrecha entre el departamento de mantenimiento y el departamento de compras, dado que los repuestos e insumos que se adquieren para mantenimiento normalmente poseen especificaciones técnicas muy definidas, que de no cumplirse a cabalidad pueden afectar seriamente el funcionamiento de las actividades de producción. 


\section{e. Informes de desempeño}

El control de las actividades de mantenimiento debe estar soportado por un sistema de información que permita la recopilación de los datos de mantenimiento y permita determinar el estado de las mismas, así como mostrar el desempeño con relación a lo planificado.

El departamento de mantenimiento debe contar con un sistema de informes regulares que reflejen el estado del mantenimiento, un resumen del trabajo de mantenimiento realizado, una lista de los gastos de mantenimiento por centro de costos o equipos importantes, informes de trabajos pendientes en términos de horas-hombre, informes de inspección del mantenimiento preventivo, tiempo muerto del equipo principal, informe de ODT's abiertas, informes de eficiencia que comparan las horas estimadas contra las horas reales, informes de variación del presupuesto mensual de mantenimiento respecto al consumo materiales y mano de obra, entre otros.

En la Tabla 5 se presentan los resultados del estudio sobre la producción de informes mensuales (casi generalizada entre las industrias) de las actividades de mantenimiento. Sin embargo, la información contenida en los informes es limitada y no genera en su totalidad información necesaria para la gestión eficiente de mantenimiento.

Los sistemas de información dan muestras de permitir un monitoreo constante de las actividades mediante el seguimiento de las ODT's, lo cual es importante para la toma de acciones correctivas sobre desviaciones en la ejecución de los trabajos. Sin embargo, el $50 \%$ de

\section{Tabla 5. Información sobre el desempeño}

\begin{tabular}{|c|c|}
\hline Característica & Porcentaje \\
\hline $\begin{array}{l}\text { Genera reportes periódicos } \\
\text { sobre la ejecución de las } \\
\text { actividades de mantenimiento. }\end{array}$ & $85,7 \%$ \\
\hline \multicolumn{2}{|l|}{$\begin{array}{l}\text { Información contenida en los } \\
\text { informes: }\end{array}$} \\
\hline - Fallas & $91,7 \%$ \\
\hline - ODT's atendidas & $66,7 \%$ \\
\hline $\begin{array}{l}\text { - Estadísticas de los tiempos } \\
\text { de operación de los equipos } \\
\text { - Estadísticas de los tiempos }\end{array}$ & $50 \%$ \\
\hline $\begin{array}{l}\text { de parada de los equipos } \\
\text { - Costo de los materiales y }\end{array}$ & $50 \%$ \\
\hline repuestos & $41,7 \%$ \\
\hline $\begin{array}{l}\text { - Costo de la mano de obra } \\
\text { - Estadísticas de los tiempos }\end{array}$ & $41,7 \%$ \\
\hline de reparación & $25 \%$ \\
\hline $\begin{array}{l}\text { Permite conocer el estatus } \\
\text { de las ODT's en cualquier }\end{array}$ & \\
\hline momento. & $50 \%$ \\
\hline $\begin{array}{l}\text { Genera indicadores de } \\
\text { desempeño. }\end{array}$ & $50 \%$ \\
\hline \multicolumn{2}{|l|}{ Indicadores generados: } \\
\hline $\begin{array}{l}\text { - Tiempo medio entre fallas } \\
\text { - Porcentaje de órdenes }\end{array}$ & $85,7 \%$ \\
\hline satisfechas a tiempo & $71,4 \%$ \\
\hline - Disponibilidad & $42,6 \%$ \\
\hline $\begin{array}{l}\text { - Productividad de la mano } \\
\text { de obra }\end{array}$ & $42,6 \%$ \\
\hline - Confiabilidad & $28,6 \%$ \\
\hline - Mantenibilidad & $14,3 \%$ \\
\hline
\end{tabular}

Fuente: Elaboración propia.

las industrias utiliza medios informales para obtener este tipo de información.

La generación de indicadores es una herramienta fundamental para la gestión de mantenimiento, proporcionando resultados principalmente de carácter económico y gerencial, que permiten observar la coherencia entre el desempeño 
Sistemas de información para la gestión de mantenimiento en la gran industria del Zulia Oliva, Karim; Arellano, Madelein; López, María y Soler, Karen

de la función de mantenimiento y los objetivos planteados por la dirección de la empresa. Como se puede observar, el $50 \%$ de las industrias genera indicadores de desempeño, entre los cuales destacan la medición del tiempo promedio entre fallas de los equipos y la satisfacción oportuna de los trabajos requeridos.

Indicadores como disponibilidad, confiabilidad y mantenibilidad propios de la gestión de mantenimiento presentan limitaciones para su elaboración debido a la deficiencia en los registros de información.

\section{f. Tecnologías de información}

Si bien no es requisito indispensable, para lograr una gestión eficiente, la incorporación al área de mantenimiento de un sistema de información asistido por computadora, su implementación facilita y simplifica significativamente el desarrollo del control de las actividades de mantenimiento.

La utilización de las tecnologías de información en este sector para el manejo y procesamiento de la información de mantenimiento se encuentra limitada, como se puede apreciar en la Tabla 6. Sólo el $35.7 \%$ de las industrias poseen sistemas automatizados, mientras que una porción similar se conduce mediante herramientas computacionales como hojas de cálculo que permiten llevar la coordinación y control de las distintas activi-

\section{Tabla 6. Tecnologías de información}

\begin{tabular}{|c|c|}
\hline Característica & Porcentaje \\
\hline $\begin{array}{l}\text { Posee un sistema de } \\
\text { información totalmente } \\
\text { automatizado } \\
\text { Posee un sistema de } \\
\text { información semi-automatizado } \\
\text { Posee un sistema de } \\
\text { información manual }\end{array}$ & $\begin{array}{l}35.7 \% \\
28.6 \%\end{array}$ \\
\hline $\begin{array}{l}\text { Tipo de sistema automatizado: } \\
\text { - Integrado } \\
\text { - Aplicación específica } \\
\text { de mantenimiento } \\
\text { - Semi-integrado }\end{array}$ & $\begin{array}{l}40 \% \\
40 \% \\
20 \% \\
\end{array}$ \\
\hline
\end{tabular}

Fuente: Elaboración propia.

dades; el $28,6 \%$ de las industrias encuestadas utiliza formatos y fichas como procedimientos manuales para estos fines.

Las industrias que utilizan sistemas automatizados integrados gestionan todas las áreas funcionales de la industria para el desarrollo de sus operaciones ${ }^{10}$. Las empresas con aplicaciones específicas de mantenimiento utilizan programas que consisten en sistemas modulares que permiten la planificación de acciones de mantenimiento, además, llevan un control sobre los activos de una empresa e interactúan únicamente con las áreas estrechamente relacionadas con el mantenimiento como lo son producción, compras, almacén y recursos humanos ${ }^{11}$. Finalmente, un $20 \%$ de las industrias repor-

10 Tales como el sistema conocido como Sistema, Aplicaciones y Productos (SAP/R3), que permite conocer la información disponible al momento y facilita el intercambio de información entre los entes involucrados.

11 Entre ellos se encuentran Máximo y Mantenimiento Preventivo. 
taron el uso de aplicaciones creadas para satisfacer los requerimientos específicos de información y que funcionan de manera aislada.

\section{Conclusiones}

Entre las industrias estudiadas se observa un alto grado de heterogeneidad en el diseño y funcionamiento de los sistemas de información de mantenimiento, que va desde sistemas sencillos con procedimientos informales, como respuesta a necesidades inmediatas, hasta sistemas más sofisticados con procedimientos sistemáticos y con un alto grado de automatización. Si bien las industrias muestran cumplimiento de las funciones de mantenimiento, sus niveles de eficiencia están estrechamente vinculados a los mecanismos de control de gestión, los cuales difieren significativamente en cantidad y en cualidad.

Los sistemas de información para mantenimiento de las grandes industrias analizadas del Estado Zulia, en general, poseen una administración adecuada de la información técnica de los equipos, mostrando deficiencias en el historial de fallas y condición operativa. Aun cuando la mayoría de las industrias funcionan con órdenes de trabajo como mecanismo de planeación y de control para los trabajos de mantenimiento, algunas emplean procedimientos pocos formales, limitando el control de la gestión. Las funciones relacionadas con las especialidades de mantenimiento y abastecimiento y control de materiales son consideradas como propias de recursos humanos y de compras o almacén de materiales, respectivamente; no se encuentra estipula- do que estas funciones son para el desarrollo de la función de mantenimiento, ocasionando riesgos de desviaciones significativas de presupuestos y fallas críticas de equipos.

Por otra parte, aunque existe una generación suficiente de reportes periódicos sobre la ejecución de las actividades de mantenimiento; sólo en la mitad de las industrias son utilizados como una herramienta para el control de gestión, con ciertas limitaciones en el tipo de estadística registrada. Indicadores propios de la función de mantenimiento aún no son registrados con regularidad en estas industrias, a pesar que un tercio de ellas utilizan sistemas totalmente automatizados.

Las deficiencias de disponibilidad de información en los sistemas de mantenimiento presentadas en estas industrias y la falta de sistemas integrados con las funciones de recursos humanos, de inventario y compras, muestran profundas brechas en la concepción de estos sistemas y se limita el control de la gestión de mantenimiento.

Algunas industrias perciben a los sistemas de mantenimiento como una herramienta de carácter utilitario para el desarrollo de las actividades cotidianas, posición cuestionable sobre la prioridad de este tipo de sistemas para las organizaciones. Otras industrias, realmente, los conciben como una forma de lograr una ventaja competitiva. Esta última decisión conlleva a una disposición hacia la mejora de los procesos en la organización. En este caso particular, las industrias con un mayor nivel de automatización mostraron sistemas de información más organizados y con mejores po- 
Sistemas de información para la gestión de mantenimiento en la gran industria del Zulia Oliva, Karim; Arellano, Madelein; López, María y Soler, Karen.

sibilidades hacia el control de la gestión de mantenimiento, lo cual muestra que las tecnologías pueden ser un elemento potencial para el desarrollo de los sistemas, pero éstos junto al accionar de los gerentes permiten, finalmente, fortalecer el desempeño de las funciones dentro de una organización.

\section{Referencias Bibliográficas}

Barros, Oscar (1998). Tecnología de la Información y su Uso en Gestión. Una visión moderna de los sistemas de información. Mcgraw-Hill Interamericana de Chile Ltda., Chile.

Bruch, John G.; Grudnitski Gary (1998). Diseño de Sistemas de Información. Editorial LIMUSA, S.A., México.

Carr, Nicholas G. (2005). Las Tecnologías de la información. ¿Son realmente una ventaja competitiva? Empresa Activa. Barcelona, España.

Cohen Karen, Daniel; Asín Lares, Enrique (2005). Sistemas de Información para los Negocios. Cuarta edición, Editorial mcgraw Hill, México.

Davis Mark M.; Aquilano, Nicholas J.; Chase Richard (2001). Fundamentos de Di- rección de Operaciones. Tercera edición Editorial mcgraw Hill, España.

Dounce, Enrique (1984). La Productividad en el Sistema Industrial. Primera edición. Compañía Industrial Continental.

Duffua, Salih; Raouf, A.; Campbell, John Dixon (2000). Sistemas de Mantenimiento. Planeación y Control. Editorial Limusa, México.

García, Francisco (1998). Implantación en SAP de la Gestión Integral de Mantenimiento. Una Aplicación Óptima. En www.mappinginteractivo. Com/plantilla-ante.asp?ld_articulo= 562. Consulta Enero 2007.

Goldratt, Eliyahu M. (1994). El Síndrome del Pajar. Ediciones Díaz Santos, Madrid.

Montilva, Jonás A. (1999). Desarrollo de los Sistemas de Información. Tercera reimpresión de la segunda edición (1992). Editorial Ingeniería, Universidad de los Andes, Consejo de Publicaciones.

O'brien, James (2001). Sistemas de Información Gerencial. Cuarta edición, Editorial mcgraw Hill, Colombia.

Oz, Effy (2001). Administración de Sistemas de Información. Segunda edición, Thomson Learning, México. 